\title{
Metastasis of Sarcomatoid Malignant Mesothelioma With p16/CDKN2A Deletion Presented as a Subcutaneous Mass in the Back: a Case Report and Review of Literature
}

\section{Kai-Bo Chen}

Zhejiang University School of Medicine Second Affiliated Hospital https://orcid.org/0000-0002-35042310

\section{Ya-Jing Huang}

the second affiliated hospital of zhejiang university, school of medicine

\section{Yi Huang}

the second affiliated hospital of zhejiang university, school of medicine

\section{Zhi-Wei Wu}

the second affiliated hospital of zhejiang university, school of medicine

Xiao-Li Jin

the second affiliated hospital of zhejiang university, school of medicine

\section{Hang Zhang}

the second affiliated hospital of zhejiang university, school of medicine

\section{Xue-Ping Xiang}

the second affiliated hospital of zhejiang university, school of medicine

\section{Lin Chen}

the second affiliated hospital of zhejiang university, school of medicine

\section{Li Chen ( $\square$ li-chen@zju.edu.cn )}

Zhejiang University School of Medicine Second Affiliated Hospital

\section{Case Report}

Keywords: Sarcomatoid, Malignant mesothelioma, Subcutaneous, p16/CDKN2A, Case report

Posted Date: December 4th, 2020

DOl: https://doi.org/10.21203/rs.3.rs-120209/v1

License: (9) This work is licensed under a Creative Commons Attribution 4.0 International License.

Read Full License 


\section{Abstract}

Background: Sarcomatoid malignant mesothelioma ( $\mathrm{MM})$ is a rare and aggressive disease, and its diagnosis is challenging.

Case presentation: A 60-year-old man was found a recurrent mass in his right back after the initial resection. A chest CT found right pleural thickening, nodular pleural thickening, pleural effusion, mediastinal and right infraclavicular lymph nodes enlargement, which indicated a right pleura MM. Immunohistochemical (IHC) stains of the resected mass showed sarcomatous atypical spindle cells, which were positive for CK $1 / 3$, CK 5/6, WT1, podoplanin and vimentin, and negative for Napsin A, TTF-1 and CDX2, and fluorescent in situ hybridization (FISH) detected homozygous $p 16 / C D K N 2 A$ deletion. The association of the chest $\mathrm{CT}$ features and the mass assessment confirmed metastatic MM in the subcutaneous layer of the back. Moreover, PET-CT showed multiple metastases in his brain. He developed massive right pleural effusion and chest tightness soon, and the mass kept growing despite of the local and systemic treatments, and he died from pulmonary failure in 3 months.

Conclusion: We described an extremely rare subcutaneous metastasis in the back from sarcomatoid MM. And we made the diagnosis by both histological and molecular analysis of the metastatic lesion. MM patients most likely have subcutaneous metastasis on head, they usually develop pleural effusion, chest pain or dyspnea, and their prognosis is very poor because of late diagnosis and insensitivity to current therapies.

\section{Background}

Malignant mesothelioma (MM) is a rare malignancy which arises from mesothelial or subthelial layer of the pleura $(80-90 \%)$, peritoneum $(10-15 \%)$, pericardium and tunica vaginalis testis $(<5 \%)$ [1]. It has been increasing over the past decade, and most cases are associated with exposure to asbestos. It is divided into three subtypes: epithelioid type, sarcomatoid type and biphasic type[2], and sarcomatoid MM is the most aggressive type. It is usually difficult to differentiate from benign mesothelial pleural proliferations or other cancers[3]. Because of its nonspecific symptom, MM is often diagnosed at late stage with distal metastases including lymph nodes, lung, liver, adrenal glands and kidney[4]. However, it is very rare to see a metastatic lesion within cutaneous or subcutaneous tissue, which is most likely via hematogenous spread. To our knowledge, only 3 such cases of sarcomatoid MM were previously reported[4-6].

\section{Case Presentation}

A 60-year-old man was referred to the General Surgery Department for a growing subcutaneous mass in the right back. He felt slight pain and discomfort when lying down. There was no local swelling or pruritus around the mass. An ultrasound showed a large tumor with hemorrhage (Diameter: $13.8 \star 4.7 \mathrm{~cm}$ ) in the muscularis. He was generally healthy and had no mental or inherited disease. He was admitted and received radical tumor resection under general anesthesia. Unexpectedly, the mass recurred quickly two 
weeks after surgery. A MRI showed tumor recurrence which intruded into thoracic cavity (Fig. 1). While, a chest $\mathrm{CT}$ found right pleural thickening, nodular pleural thickening, pleural effusion, mediastinal and right infraclavicular lymph nodes enlargement (Fig. 2A and 2B). And a needle biopsy of the right pulmonary nodule was performed, which reported few atypical cells.

The histological features of the resected mass revealed that sarcomatous atypical spindle cells with enlarged and elongated nuclei were arranged in fascicles. Immunohistochemical (IHC) stains were positive for cytokeratin (CK) $1 / 3$, CK $5 / 6$, WT1, podoplanin and vimentin, with focal positivity of CAM 5.2, and negative for carcinoma markers, including Napsin A, thyroid transcription factor 1 (TTF)-1 and caudal-related homeobox gene 2 (CDX2) (Fig. 3). Calretinin was not found expressed in this case. Myogenic marker desmin was also negatively expressed. Ki-67 index was 80\% (Fig. 3). Fluorescent in situ hybridization (FISH) in cytology specimens showed homozygous p16/CDKN2A deletion (Fig. 4). Combined with clinical data, the histopathological diagnosis supported sarcomatoid MM with subcutaneous metastasis.

By a multi-disciplinary team (MDT) discussion, high-intensity focused ultrasound (HIFU) was used to treat the local recurrence. Meanwhile, one course of albumin paclitaxel (300 mg d1) and cisplatin (35 mg d1-3) was intravenously administrated. 2 week later, a PET-CT found that there was necrosis in the center of the subcutaneous mass, the right pulmonary nodule was as before, the mediastinal and right infraclavicular lymph nodes were smaller, but multiple metastases were seen in the brain (Fig. 5).

Afterwards, radiation was also performed on the mass. But the patient felt aggravating chest tightness and mild pain on the back during this period. Another chest CT found massive right pleural effusion. So pleural aspiration drainage was done to relieve the symptom, approximately $2000 \mathrm{ml}$ of hemorrhagic pleural effusion was drained from the chest cavity. The tumor markers of the pleural effusion were elavated: CA-123 1456.1 U/ml, CA-211 $6.8 \mathrm{ng} / \mathrm{ml}$. Twice PD-1 inhibitor (Camrelizumab) $(200 \mathrm{mg}$ ) was administrated, and three times intrapleural chemotherapy of cisplatin $(40 \mathrm{mg})$ was done. 1 month later, a chest CT showed that right pulmonary nodule was stable, the mediastinal and right infraclavicular lymph nodes decreased, but the tumor in the back still significantly increased (Figure Fig. 2D and 2D). The patient discharged during the chemotherapy interval. However, he got worse soon and ultimately succumbed to pulmonary failure in 1 month after discharge.

\section{Discussion}

$\mathrm{MM}$ a rare malignant disease, and its diagnosis is usually challenging for pathologists[7]. Because its phenotype varies among patients, and it mimics benign reactive mesothelial proliferations and other cancers. In many cases, only cytological material or a limited amount of tissue is available for pathologic evaluation [8]. Sometimes, video-assisted thoracoscopic surgery (VATS) is necessary for a deep and large biopsy and assessment of its respectability $[9,10]$. The International Mesothelioma Interest Group recommends that at least 2 mesothelial (calretinin, CK 5 or 5/6, WT1 and podoplanin) and 2 carcinoma markers (Claudin 4, MOC31, CEA, BER-EP4, BG8, TTF-1 and Napsin A) for IHC differential diagnosis[11]. 
Recent studies showed that BAP1 mutations and $p 16$ deletions by FISH were reliable in differentiating malignant mesotheliomas from benign reactive mesothelial proliferations[7, 12]. Several studies have reported $p 16 / C D K N 2 A$ deletions in up to $80 \%$ of primary MM, depending on the histologic subtype (90$100 \%$ of sarcomatoid type; $70 \%$ of epithelioid and biphasic types)[13]. A study reported that P16 immunoreactivity in stromal cells of MM had significant relation with the patients' high exposure to asbestos[14]. And the presence of a $p 16$ homozygous deletion correlates with shorter survival in patients with MM[15]. Recently, circulating miRNAs and extracellular vesicles (EVs) are discovered to be novel and non-invasive biomarkers for early detection, differential diagnosis and predicting prognosis of $M M[16$, 17]. The resected tumor specimen of this case was positive for CK5/6, WT 1 and podoplanin, and negative for Naspin A and TTF-1, which meets the criteria above. Also, the pathological features of sarcomatous atypical spindle cells of this case indicated sarcomatous type of MM. Further FISH detected homozygous $p 16 / C D K N 2 A$ deletion. This result demonstrates that a metastatic mass is also a reliable diagnostic tissue in the intractable MM case, which has not been verified in the previous studies.

CT features of MM include unilateral pleural effusion, nodular pleural thickening, interlobar fissure thickening, calcified pleural plaques, chest wall involvement and hilar and mediastinal lymph nodes enlargement[18]. However, its sensitivity and specificity are only 70\%[19]. MRI can provide additional information of invasive growth of MM such as invasion of the diaphragm, endothoracic fascia and chest wall[20]. PET-CT can identify MM from benign disease, but not metastatic pleural malignancy[21]. And PET-CT is able to indentify nodal and extrathoracic metastases. A study found that Mean SUVmax value was higher in sarcomatoid $(11.8 \pm 4.6)$ and biphasic type $(9.3 \pm 7.0)$ rather than in epithelioid type $(6.9 \pm$ 3.8) $(P<0.01)$, and high SUVmax values were significantly associated with a worse prognosis[22]. We found brain metastases by PET-CT, and the SUVmax of the right lung nodule in this case was as high as 19.0 , which indicated highly malignancy.

It is common to see lymph nodes and organ involvement. As we noticed, there were lymph nodes, subcutaneous and brain metastases in this case. The disseminating routes of MM are direct invasion, lymphatic and hematogenous spread[23]. The solitary mass in the back was probably via hematogenous in this case. To our knowledge, there are 18 case reports of cutaneous or subcutaneous metastases from MM [4-6, 23-36] (Fig. 6), but only 3 sarcomatoid type of MM among them[4-6]. The data of the previous researches indicated that the most common cutaneous or subcutaneous metastases from MM occurred on head, chest pain or dyspnea was a main symptom, about half of the patients suffered pleural effusion, they usually received chemotherapy, and 1-year-survival rate was only $20 \%$ (Fig. 6).

The majority of patients with MM develop breathlessness with pleural effusion[37]. Therapeutic pleural aspiration and chemical pleurodesis are two ways to control pleural fluid and improve patients' quality of life[37]. Pleural aspiration and intrapleural chemotherapy of cisplatin was done in this case, but its effectiveness of intrapleural cisplatin was uncertain since the follow-up was too short.

Generally, MM is incurable disease, the median survival period is less than 1 year[38], and the 5-yearsurvival rate is only $5 \%[39]$. A sarcomatoid type is associated with a worst outcome, with a median 
survival of just 4 months[37]. This case was found multiple distant metastases and deteriorated very quickly, and the patient died within 3 months since initial diagnosis. Surgery is limited only for MM patients in early stage. Based on our experience, local resection of a subcutaneous metastatic lesion was not a good option, since it turned out a quick recurrence. A needle biopsy might be worthy doing for diagnosis. HIFU therapy rather than radiation showed a therapeutic potential. Systemic treatment of chemotherapy and immunotherapy are delivered on the patient, but MM is highly resistant to them specially the metastastic lesion. A small number of studies showed that pemetrexed/raltitrexed plus cisplatin/carboplatin could achieve better response rates[40, 41]. PD-L1 expression is relatively common (>30\%) for MM samples[42], and it is related to poor prognosis in MM patients[43]. Immunotherapy such as nivolumab is being on clinical trials as a second or third line treatment for $\operatorname{MM}[2,44]$. In this case, the mediastinal and right infraclavicular lymph nodes seemed sensitive to cytotoxic agents, however, the pulmonary nodule and the subcutaneous mass were both resistant to the agents. Hence, more clinical trials focusing on effective agents should be conducted in the future.

\section{Conclusion}

In conclusion, we presented an extremely rare case of a subcutaneous metastatic mass from right pleural sarcomatoid MM. We made the diagnosis by both histological and molecular analysis of the subcutaneous lesion instead of a limited amount of lung tissue biopsy. PET-CT was useful to indentify extrathoracic metastases. Besides, MM patients most likely have subcutaneous metastasis on head, they usually develop pleural effusion, chest pain or dyspnea, and their prognosis is very poor because of late diagnosis and insensitivity to chemotherapy or other treatments.

\section{Abbreviations}

MM: malignant mesothelioma; FISH: fluorescent in situ hybridization; MDT: multi-disciplinary team; HIFU: high-intensity focused ultrasound; IHC: Immunohistochemical; CK: cytokeratin; TTF-1: thyroid transcription factor 1; CDX2: caudal-related homeobox gene 2; VATS: video-assisted thoracoscopic surgery; EVs: extracellular vesicles.

\section{Declarations}

\section{Acknowledgements}

We would like to thank Cong-Cong Yu and Jia-Ning Chen for their supports.

\section{Authors' contributions}

$\mathrm{KBC}, \mathrm{YJH}$ performed histological evaluation, made the pathological diagnosis and drafted the manuscript. YJH performed the immunohistochemistry. GPX performed the FISH examination. LinC provided PET-CT images. KBC, XLJ, HZ edited the images and tables. YH, ZWW, LC advised on 
manuscript preparation and made revision to the final manuscript. All authors read and approved the final manuscript.

\section{Funding}

This work was supported by the Basic Public Welfare Research Project of Zhejiang Province (No. LGD19H160004 and No. LGF21H030005).

\section{Availability of data and materials}

The dataset supporting the conclusion of this article is included within the article.

\section{Ethics approval and consent to participate}

This work has been approved by Medical Ethics Committees of the Second Affiliated Hospital of Zhejiang University, School of Medicine (approval number is 2020-514), and informed consent form has been obtained from a relative of the patient.

\section{Consent for publication}

Not applicable.

\section{Competing interests}

The authors declare that they have no competing interests.

\section{References}

1. Micolucci L, Akhtar MM, Olivieri F, et al. Diagnostic value of microRNAs in asbestos exposure and malignant mesothelioma: systematic review and qualitative meta-analysis. Oncotarget. 2016;7(36):58606-58637.

2. Brcic L, Kern I. Clinical significance of histologic subtyping of malignant pleural mesothelioma. Translational Lung Cancer Research. 2020;9(3):924-933.

3. Bruno R, Ali G, Fontanini G. Molecular markers and new diagnostic methods to differentiate malignant from benign mesothelial pleural proliferations: a literature review. J Thorac Dis. 2018;10(Suppl 2):S342-S352.

4. Elbahaie AM, Kamel DE, Lawrence J, et al. Late cutaneous metastases to the face from malignant pleural mesothelioma: A case report and review of the literature. World Journal of Surgical Oncology. 2009;7:5.

5. Patel T, Bansal R, Trivedi P, et al. Subcutaneous metastases of sarcomatoid mesothelioma with its differential diagnosis on fine needle aspiration--a case report. Indian journal of pathology \& microbiology. 2005;48(4):482-484. 
6. Krishnaraj N, Leen GLS, Kane P, et al. Malignant mesothelioma presenting as stroke - a case report. European Journal of Cancer Care. 2003;12(4):365-368.

7. Ali G, Bruno R, Fontanini G. The pathological and molecular diagnosis of malignant pleural mesothelioma: a literature review. J Thorac Dis. 2018;10(Suppl 2):S276-S284.

8. Monaco S, Mehrad M, Dacic S. Recent Advances in the Diagnosis of Malignant Mesothelioma: Focus on Approach in Challenging Cases and in Limited Tissue and Cytologic Samples. Advances in Anatomic Pathology. 2018;25(1):24-30.

9. Perikleous P, Waller DA. Video assisted thoracoscopic and open chest surgery in diagnosis and treatment of malignant pleural diseases. Journal of visualized surgery. 2017;3:85-85.

10. Hasegawa S, Kondo N, Matsumoto S, et al. Practical approaches to diagnose and treat for T0 malignant pleural mesothelioma: a proposal for diagnostic total parietal pleurectomy. Int $\mathrm{J}$ Clin Oncol. 2012;17:33-39.

11. Klebanov N, Reddy BY, Husain S, et al. Cutaneous Presentation of Mesothelioma With a Sarcomatoid Transformation. Am J Dermatopathol. 2018;40(5):378-382.

12. Hjerpe A, Abd Own S, Dobra K. Integrative approach to cytologic and molecular diagnosis of malignant pleural mesothelioma. Transl Lung Cancer Res. 2020;9(3):934-943.

13. Husain AN, Colby TV, Ordonez NG, et al. Guidelines for Pathologic Diagnosis of Malignant Mesothelioma 2017 Update of the Consensus Statement From the International Mesothelioma Interest Group. Arch Pathol Lab Med. 2018;142(1):89-108.

14. Kettunen E, Savukoski S, Salmenkivi K, et al. CDKN2A copy number and p16 expression in malignant pleural mesothelioma in relation to asbestos exposure. BMC Cancer. 2019;19(1):507.

15. Lopez-Rios F, Chuai S, Flores R, et al. Global gene expression profiling of pleural mesotheliomas: Overexpression of aurora kinases and P16/CDKN2A deletion as prognostic factors and critical evaluation of microarray-based prognostic prediction. Cancer Research. 2006;66(6):2970-2979.

16. Sturchio E, Berardinelli MG, Boccia P, et al. MicroRNAs diagnostic and prognostic value as predictive markers for malignant mesothelioma. Arch Environ Occup Health. 2020:1-12.

17. Ahmadzada T, Kao S, Reid G, et al. Extracellular vesicles as biomarkers in malignant pleural mesothelioma: A review. Crit Rev Oncol Hematol. 2020;150:102949.

18. Wang ZJ, Reddy GP, Gotway MB, et al. Malignant Pleural Mesothelioma: Evaluation with CT, MR Imaging, and PET. RadioGraphics. 2004;24(1):105-119.

19. Rahman NM, Pepperell J, Rehal S, et al. Effect of Opioids vs NSAIDs and Larger vs Smaller Chest Tube Size on Pain Control and Pleurodesis Efficacy Among Patients With Malignant Pleural Effusion The TIME1 Randomized Clinical Trial. Jama-Journal of the American Medical Association. 2015;314(24):2641-2653.

20. RT H, VW R, CB B, et al. Staging of malignant pleural mesothelioma: comparison of CT and MR imaging. AJR. 1999;172:1039-1047. 
21. Benard F, Sterman D, Smith RJ, et al. Metabolic imaging of malignant pleural mesothelioma with fluorodeoxyglucose positron emission tomography. Chest. 1998;114(3):713-722.

22. Lococo F, Rena O, Torricelli F, et al. F-18-fluorodeoxyglucose positron emission tomography in malignant pleural mesothelioma: diagnostic and prognostic performance and its correlation to pathological results. Interactive Cardiovascular and Thoracic Surgery. 2020;30(4):593-596.

23. Ward RE, Ali SA, Kuhar M. Epithelioid malignant mesothelioma metastatic to the skin: A case report and review of the literature. J Cutan Pathol. 2017;44(12):1057-1063.

24. Terada T. Skin Metastasis of Pleural Epithelioid Malignant Mesothelioma. Applied Immunohistochemistry \& Molecular Morphology. 2011;19(1):92-93.

25. Edstrom LE, Dawson PJ, Kohler J, et al. MALIGNANT MESOTHELIOMA - A METASTASIS TO THE FACE. Journal of Surgical Oncology. 1980;14(3):251-254.

26. Pierard GE. CUTANEOUS METASTASES OF MALIGNANT MESOTHELIOMA. Archives of Dermatology. 1984;120(12):1557-1557.

27. Dutt PL, Baxter JW, Omalley FP, et al. DISTANT CUTANEOUS METASTASIS OF PLEURAL MALIGNANT MESOTHELIOMA. Journal of Cutaneous Pathology. 1992;19(6):490-495.

28. Grellner W, Staak M. MULTIPLE SKELETAL-MUSCLE METASTASES FROM MALIGNANT PLEURAL MESOTHELIOMA. Pathology Research and Practice. 1995;191(5):456-460.

29. Laurini JA, Castiglioni T, Elsner B, et al. Soft tissue metastasis as initial manifestation of pleural malignant mesothelioma - A case report. International Journal of Surgical Pathology. 1999;7(1):3944.

30. Parra HS, Zucali PA, Colombo P, et al. Uncommon manifestations of common malignancies - Case 1. Soft-tissue metastases from malignant pleural mesothelioma. Journal of Clinical Oncology. 2004;22(15):3191-3192.

31. Maiorana A, Giusti F, Cesinaro AM, et al. Cutaneous metastases as the first manifestation of pleural malignant mesothelioma. Journal of the American Academy of Dermatology. 2006;54(2):363-365.

32. Kanbay A, Oguzulgen Kl, Ozturk C, et al. Malignant pleural mesothelioma with scalp, cerebellar, and finger metastases: A rare case. Southern Medical Journal. 2007;100(1):63-65.

33. Tertemiz KC, Ozgen Alpaydin A, Gurel D, et al. Multiple distant metastases in a case of malignant pleural mesothelioma. Respiratory medicine case reports. 2014;13:16-18.

34. Beer TW, Heenan PJ. Malignant mesothelioma presenting as a lip tumor: Report of two cases with one unrecognized by 166 pathologists. American Journal of Dermatopathology. 2007;29(4):388-391.

35. Cassarino DS, Xue W, Shannon KJ. Widespread cutaneous and perioral metastases of mesothelioma. Journal of Cutaneous Pathology. 2003;30(9):582-585.

36. Mueller CSL, Reichrath J, Tilgen W. Disseminated cutaneous metastasis of a biphasic pleural mesothelioma. Journal of the European Academy of Dermatology and Venereology. 2009;23(2):226227. 
37. Bibby AC, Tsim S, Kanellakis N, et al. Malignant pleural mesothelioma: an update on investigation, diagnosis and treatment. Eur Respir Rev. 2016;25(142):472-486.

38. Kindler HL. Systemic Treatments for Mesothelioma: Standard and Novel. Current Treatment Options in Oncology. 2008;9(2-3):171-179.

39. Neumann V, Gunther S, Muller KM, et al. Malignant mesothelioma - German mesothelioma register 1987-1999. International Archives of Occupational and Environmental Health. 2001;74(6):383-395.

40. Vogelzang NJ, Rusthoven JJ, Symanowski J, et al. Phase III study of pemetrexed in combination with cisplatin versus cisplatin alone in patients with malignant pleural mesothelioma. Journal of Clinical Oncology. 2003;21(14):2636-2644.

41. Meerbeeck JV, Manegold C, Gaafar R, et al. A randomized phase III study of cisplatin with or without raltitrexed in patients (pts) with malignant pleural mesothelioma (MPM): an intergroup study of the EORTC Lung Cancer Group and NCIC. Paper presented at: Central European Lung Cancer Conference2004.

42. Brosseau S, Danel C, Scherpereel A, et al. Shorter Survival in Malignant Pleural Mesothelioma Patients With High PD-L1 Expression Associated With Sarcomatoid or Biphasic Histology Subtype: A Series of 214 Cases From the Bio-MAPS Cohort. Clinical Lung Cancer. 2019;20(5):E564-E575.

43. Nguyen BH, Montgomery R, Fadia M, et al. PD-L1 expression associated with worse survival outcome in malignant pleural mesothelioma. Asia-Pacific Journal of Clinical Oncology. 2018;14(1):69-73.

44. Scherpereel A, Mazieres J, Greillier L. Nivolumab or nivolumab plus ipilimumab in patients with relapsed malignant pleural mesothelioma (IFCT-1501 MAPS2): a multicentre, open-label, randomised, non-comparative, phase 2 trial. Lancet Oncology. 2019;20(3):E132-E132.

\section{Figures}




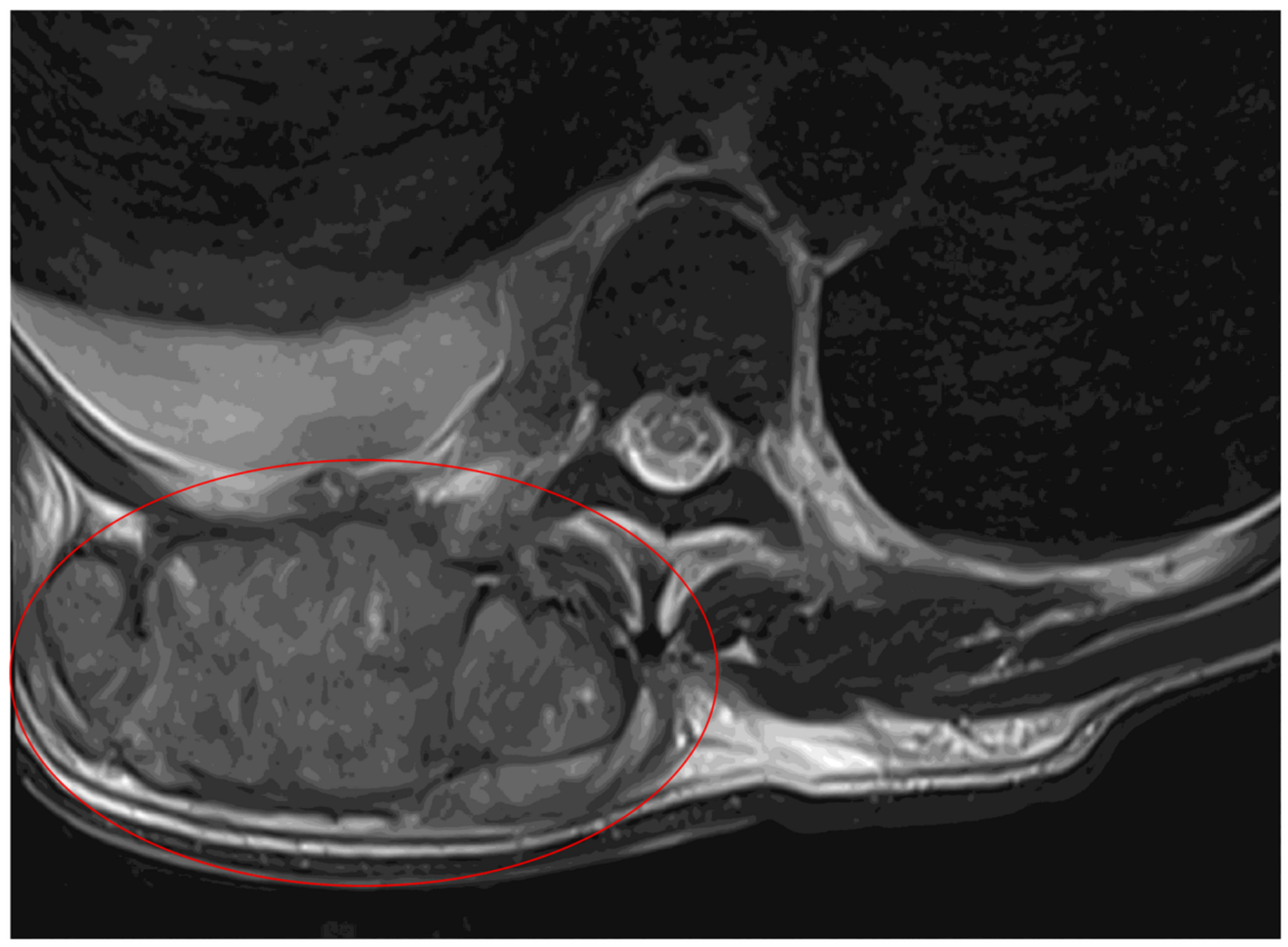

\section{Figure 1}

MRI showed an irregular tumor in the back which intruded into the thoracic cavity. 


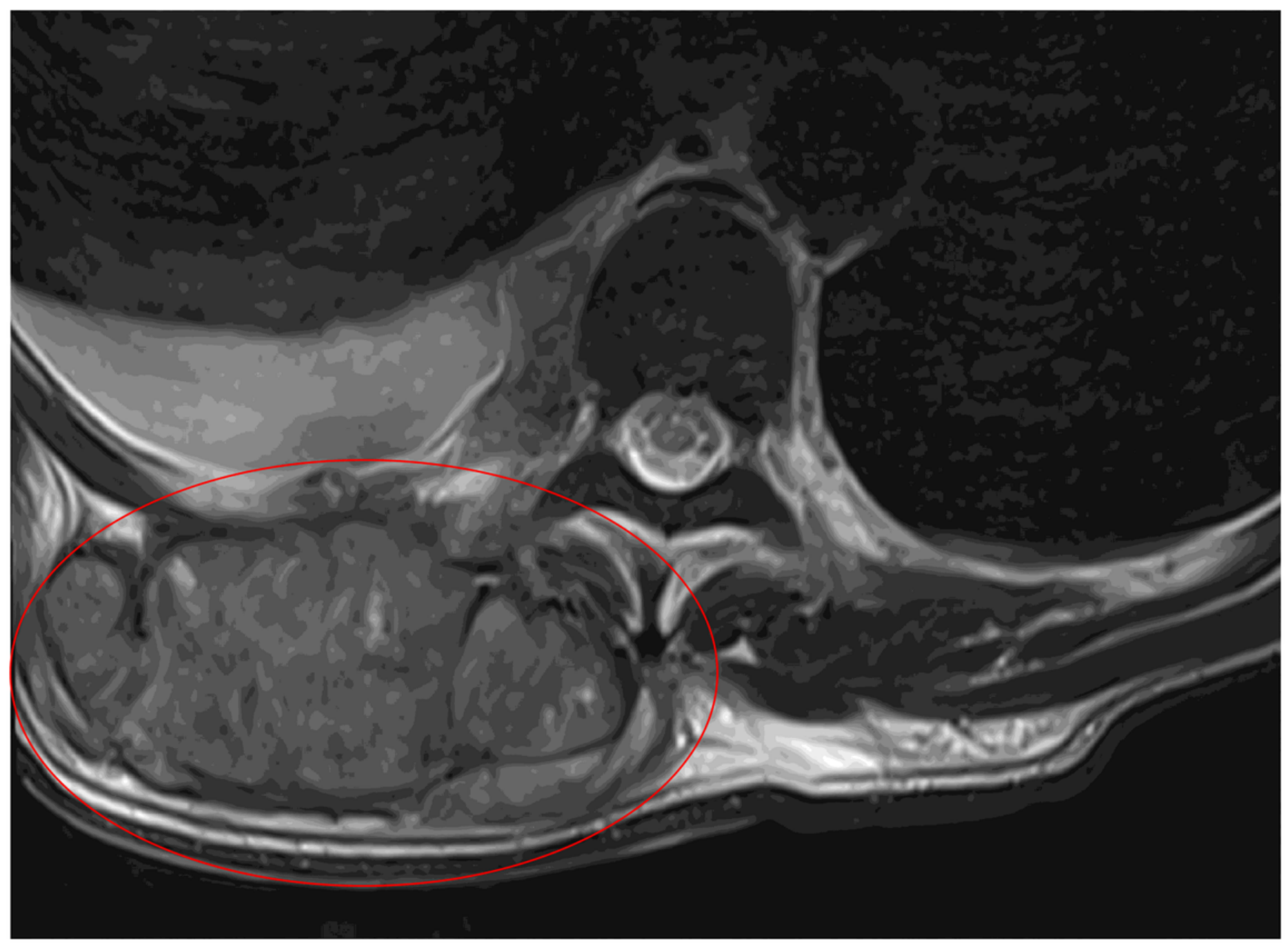

\section{Figure 1}

MRI showed an irregular tumor in the back which intruded into the thoracic cavity. 


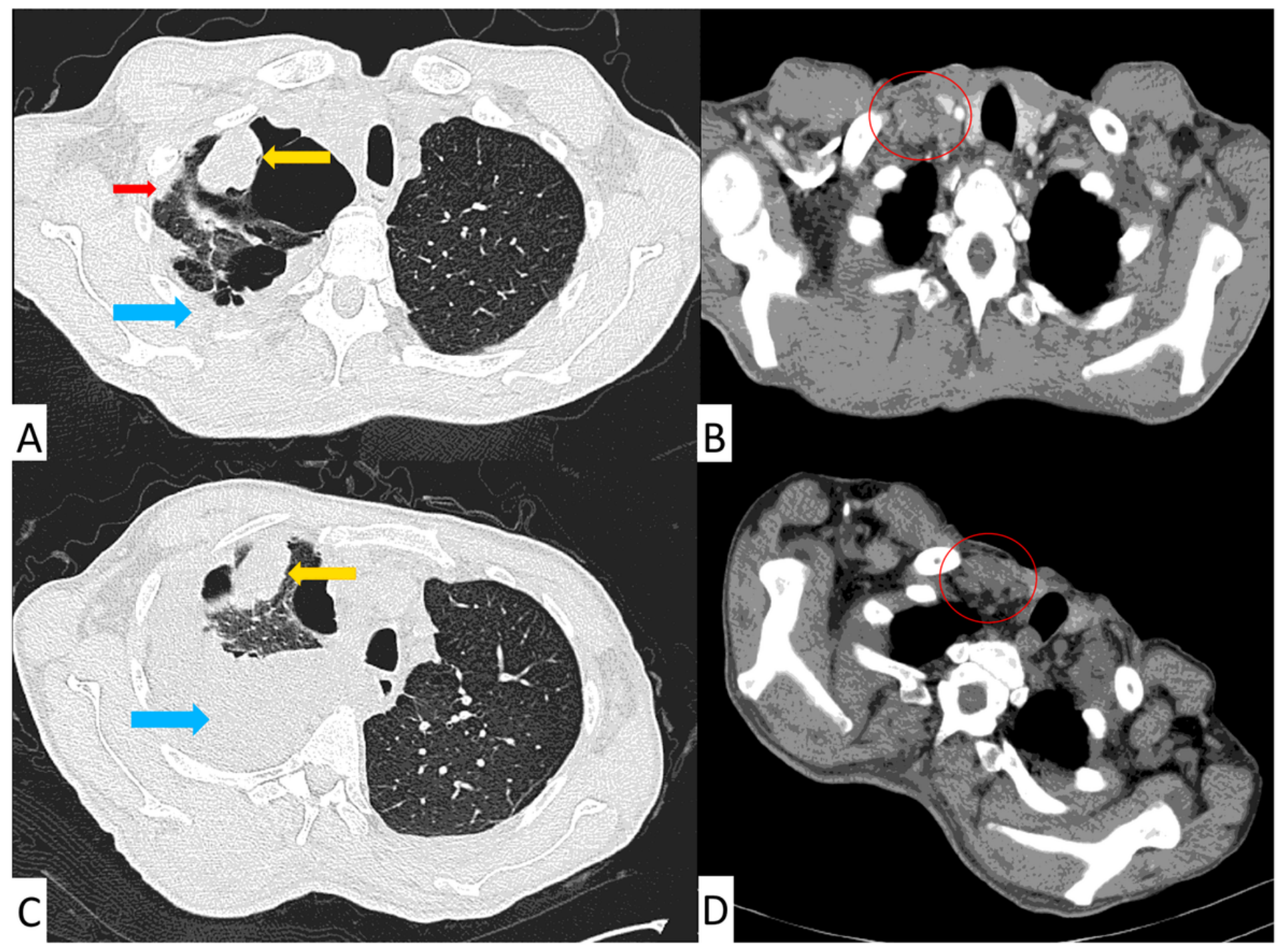

Figure 2

A. CT showed right pleural thickening (red arrow), nodular pleural thickening (yellow arrow) and mild pleural effusion (blue arrow). B. right infraclavicular lymph nodes enlargement. C. the nodular pleural thickening (yellow arrow) was stable and the pleural effusion (blue arrow) increased after treatment. D. the right infraclavicular lymph nodes decreased after treatment. 


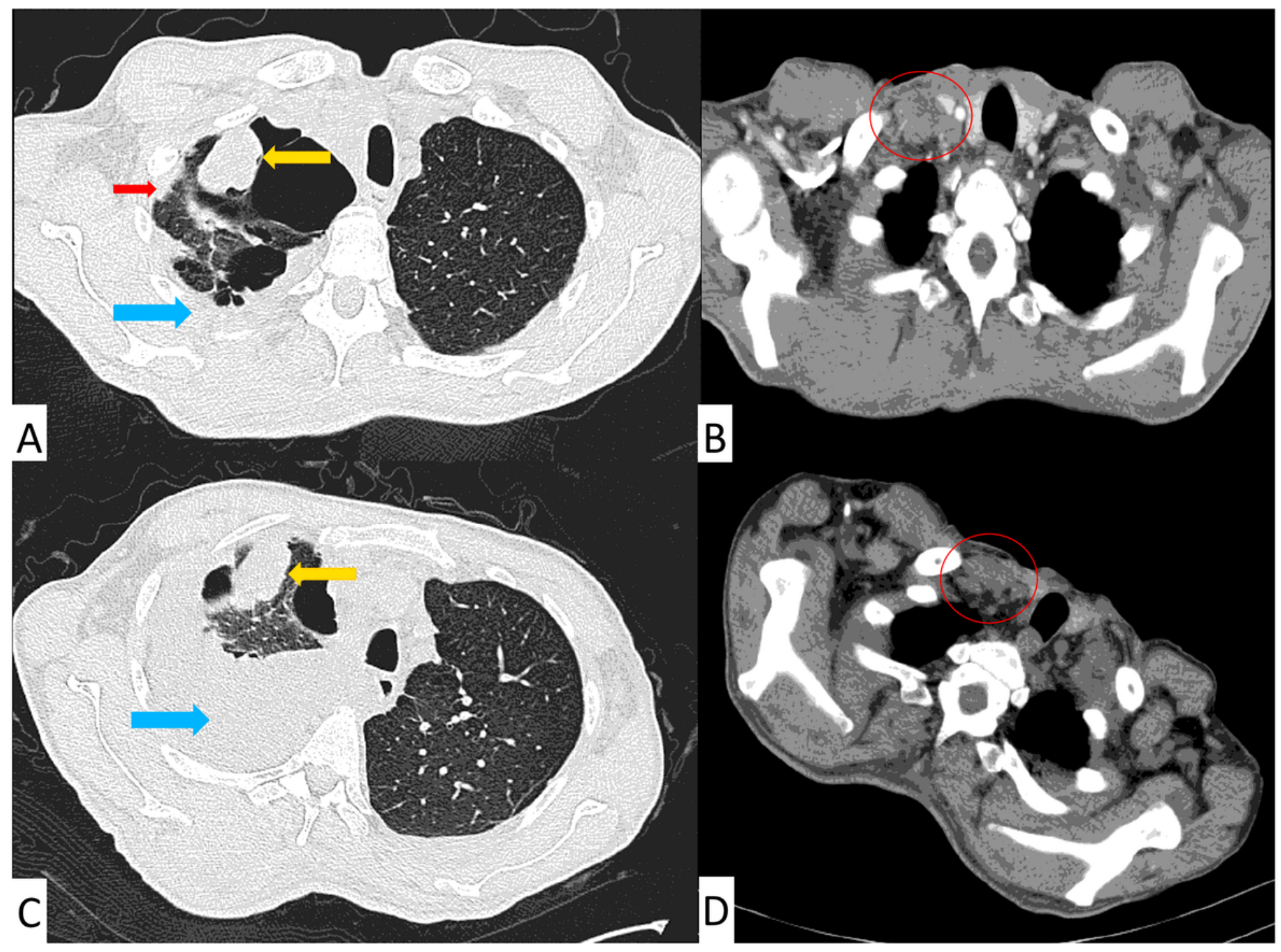

Figure 2

A. CT showed right pleural thickening (red arrow), nodular pleural thickening (yellow arrow) and mild pleural effusion (blue arrow). B. right infraclavicular lymph nodes enlargement. C. the nodular pleural thickening (yellow arrow) was stable and the pleural effusion (blue arrow) increased after treatment. D. the right infraclavicular lymph nodes decreased after treatment. 


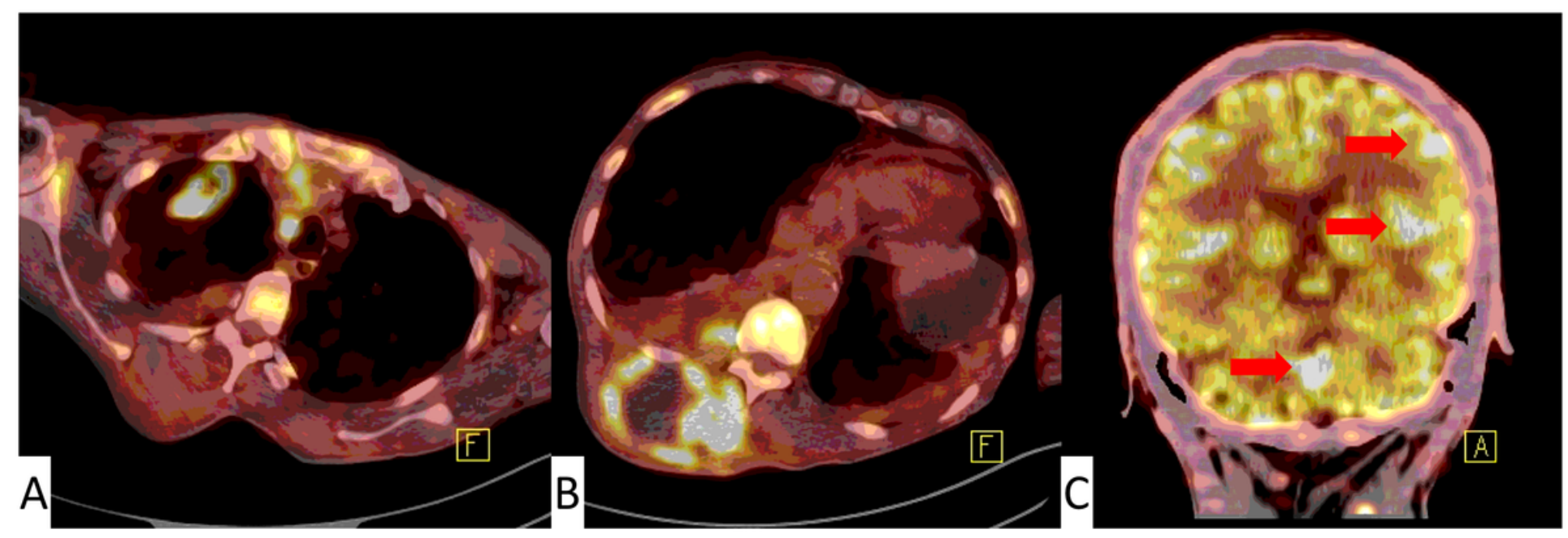

\section{Figure 3}

Microscopic features of the subcutaneous mass in this case (Magnification $\times 200)$. A. Hematoxylin-eosin staining of tumor specimen showed sarcomatous atypical spindle cells with enlarged and elongated nuclei were arranged in fascicles. B. CK(cytokeratin) $1 / 3$ positive. C. CK5/6 positive D. Wilm's tumor 1 (WT)-1 postive E. Podoplanin postive F. Vimentin positive G. Napsin A negative H. Thyroid transcription factor 1 (TTF)-1 negative I. Ki-67 index was 80\%.

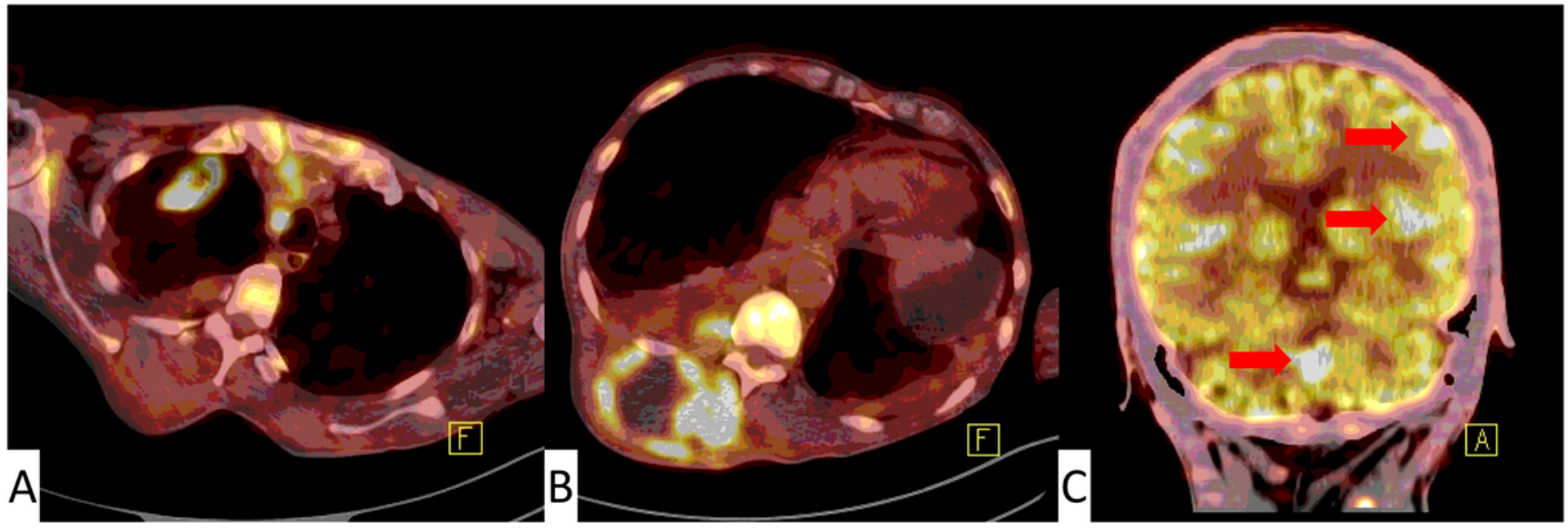

\section{Figure 3}

Microscopic features of the subcutaneous mass in this case (Magnification $\times 200)$. A. Hematoxylin-eosin staining of tumor specimen showed sarcomatous atypical spindle cells with enlarged and elongated nuclei were arranged in fascicles. B. CK(cytokeratin) 1/3 positive. C. CK5/6 positive D. Wilm's tumor 1 (WT)-1 postive E. Podoplanin postive F. Vimentin positive G. Napsin A negative H. Thyroid transcription factor 1 (TTF)-1 negative I. Ki-67 index was $80 \%$. 

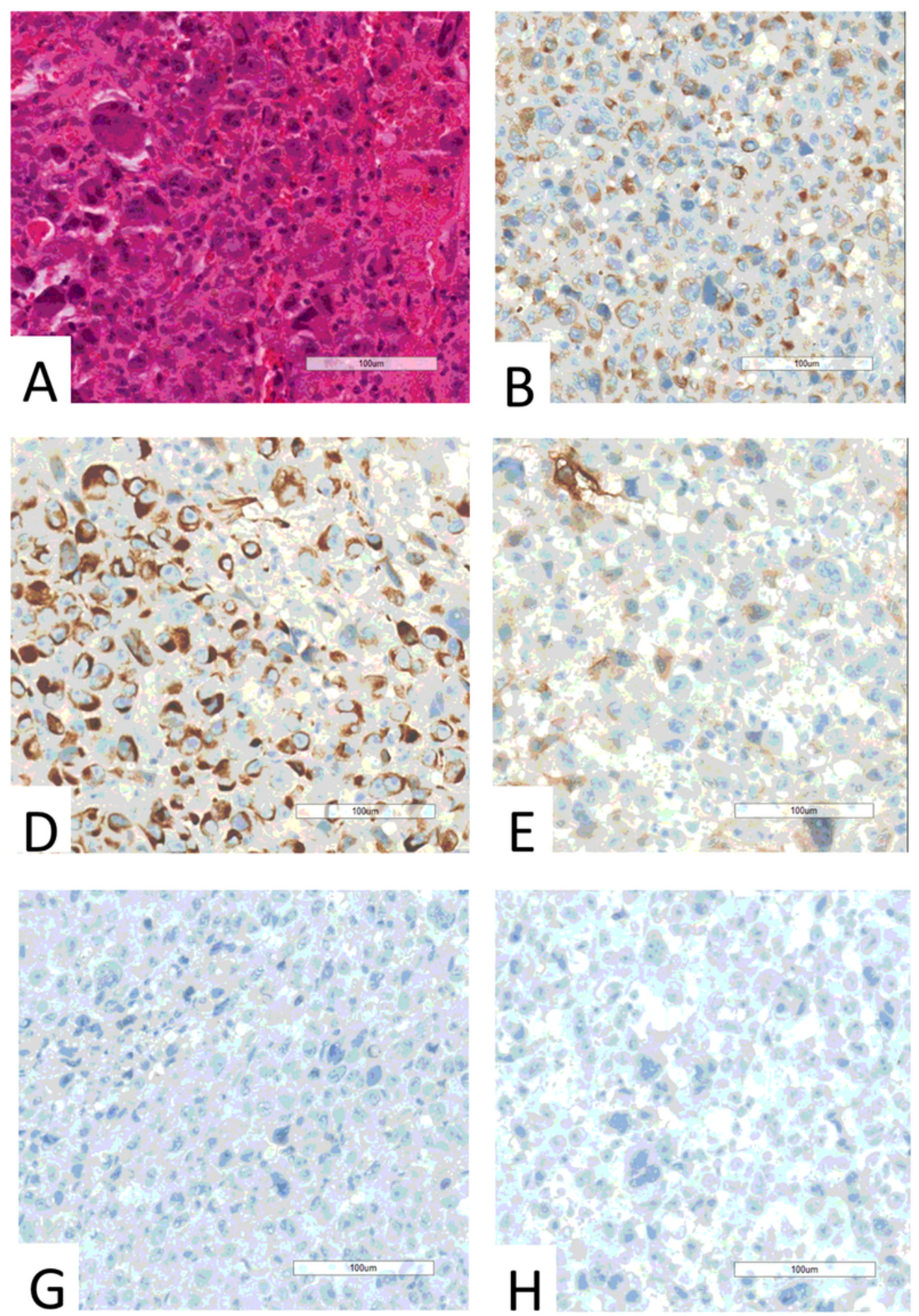
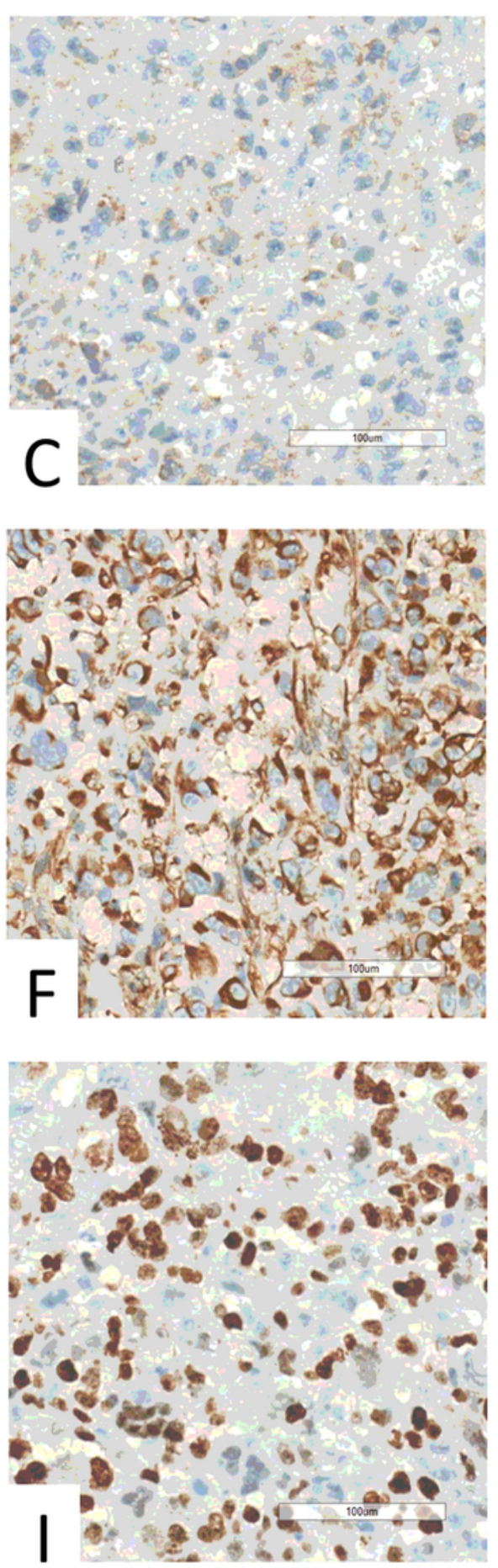

\section{Figure 4}

Fluorescent in situ hybridization (FISH) in cytology specimens showed p16 deletion (magnification $\times 1000$ ): only 2 green signals (9p centromere) and no red signal ( $p 16)$. 

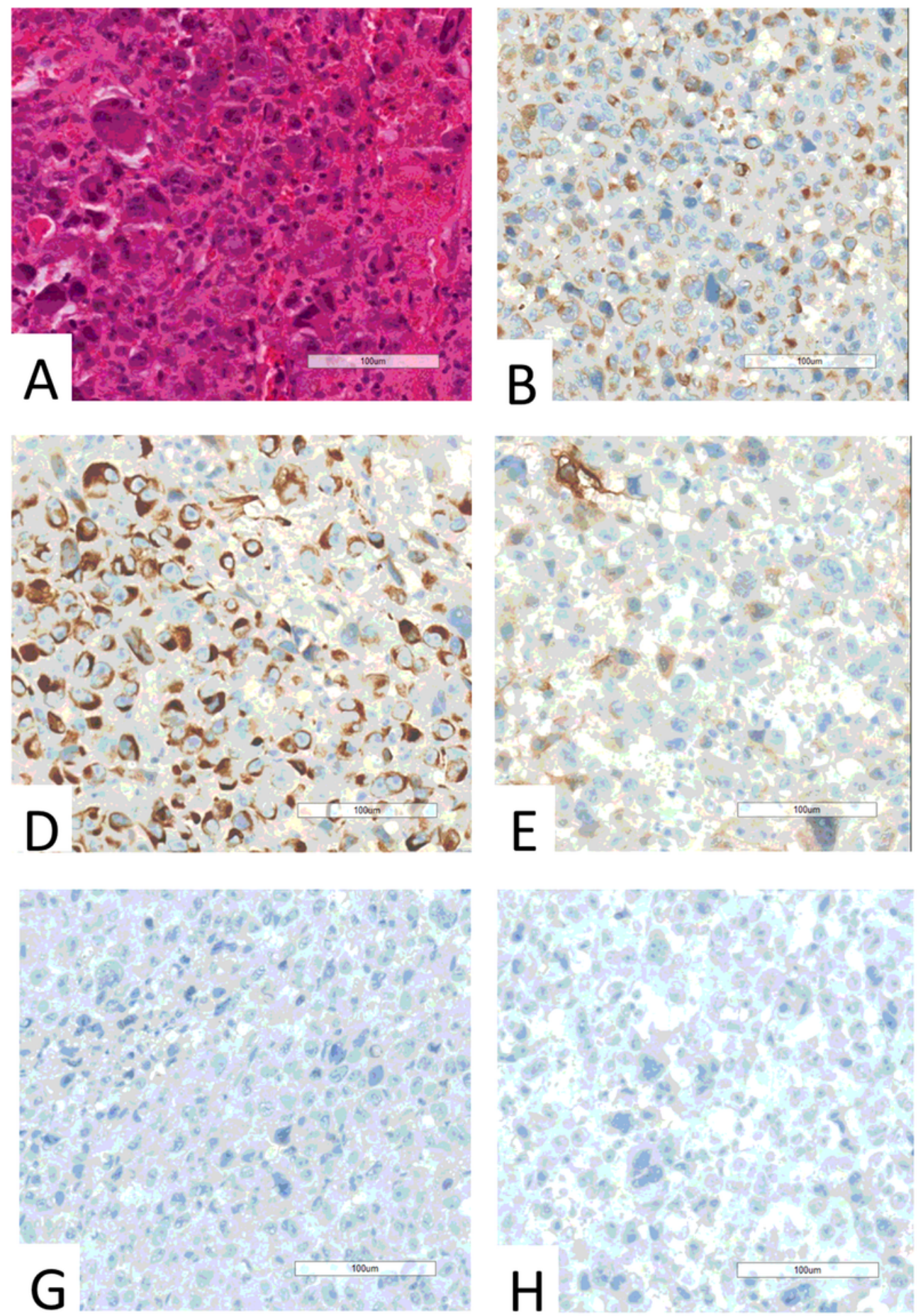
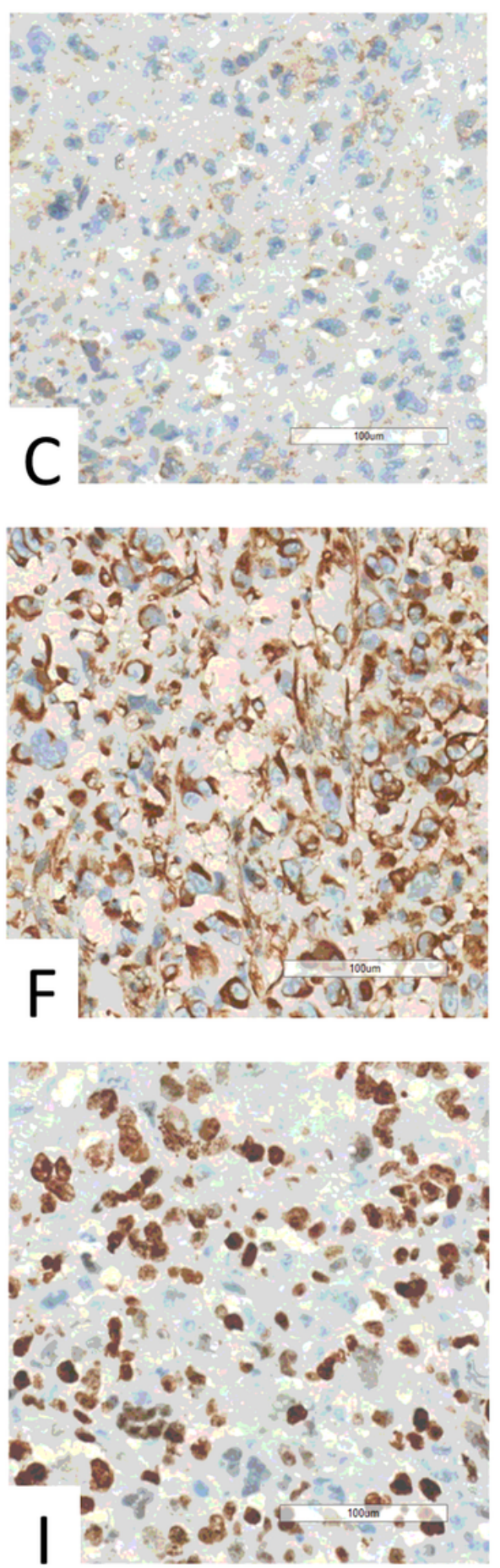

\section{Figure 4}

Fluorescent in situ hybridization (FISH) in cytology specimens showed p16 deletion (magnification $\times 1000$ ): only 2 green signals (9p centromere) and no red signal ( $p 16)$. 


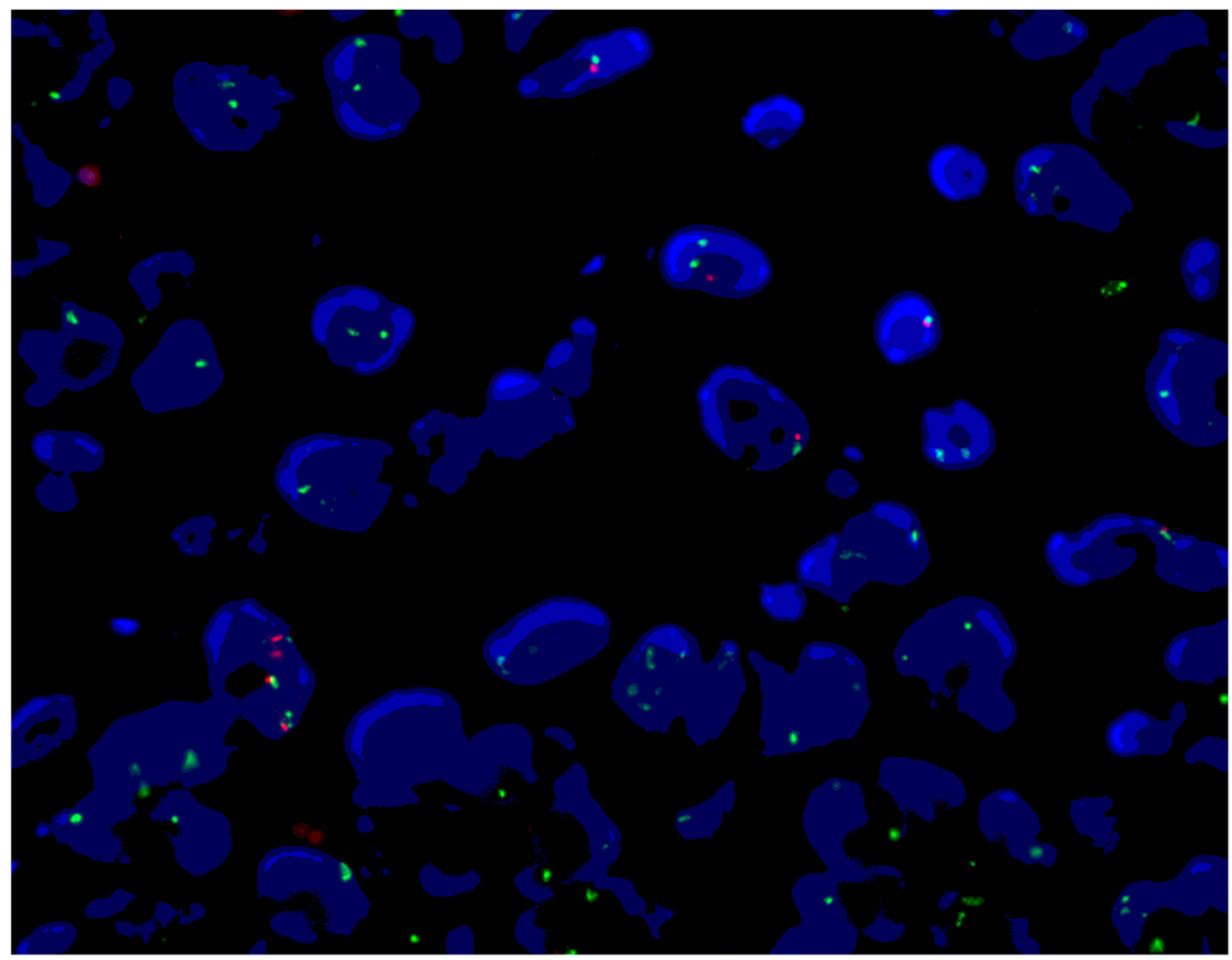

Figure 5

PET-CT found that there was necrosis in the center of the mass in the back, the right pulmonary nodule was as before, the right infraclavicular lymph nodes were smaller, and multiple metastases were seen in the brain. 


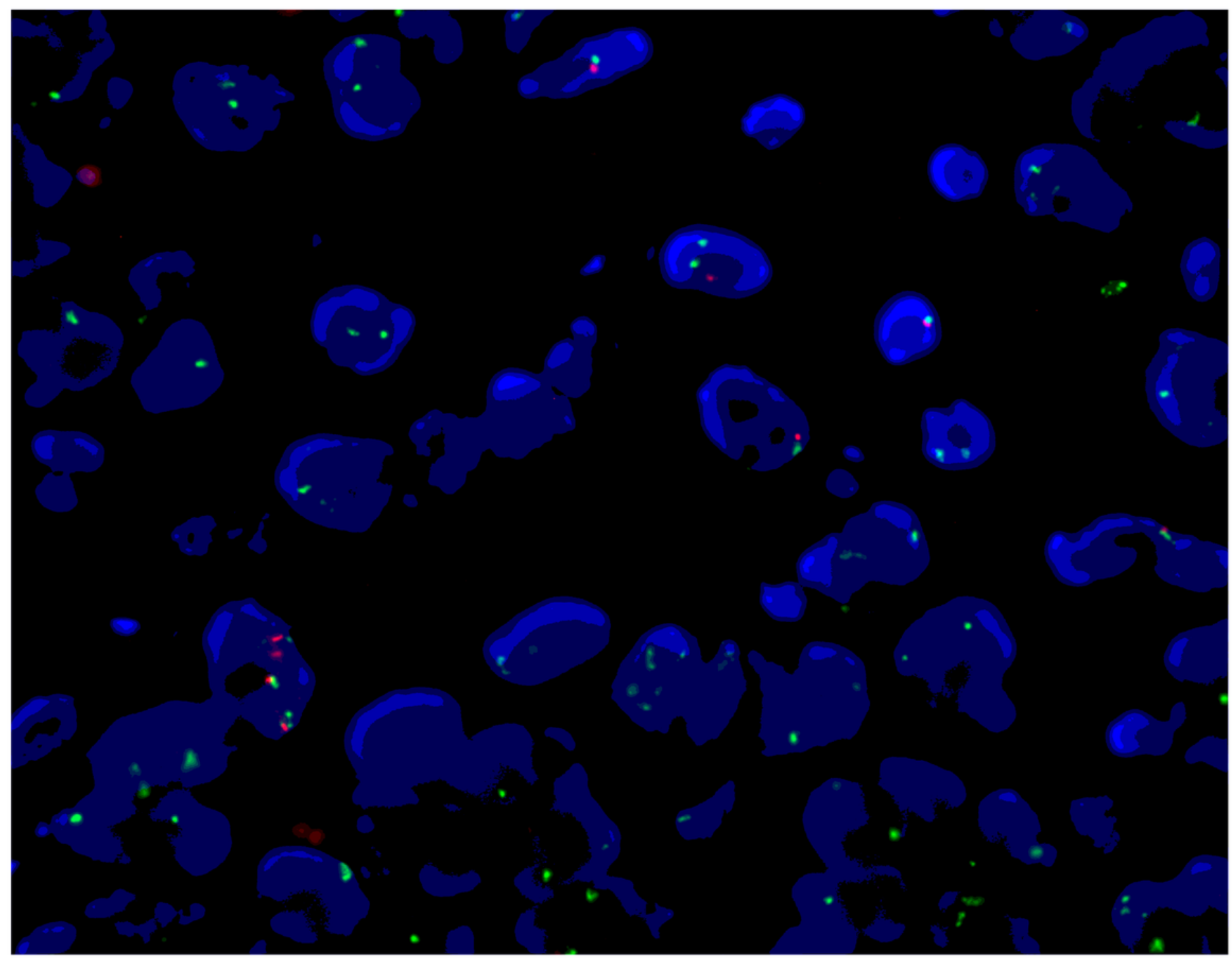

Figure 5

PET-CT found that there was necrosis in the center of the mass in the back, the right pulmonary nodule was as before, the right infraclavicular lymph nodes were smaller, and multiple metastases were seen in the brain. 


\section{8 cases}

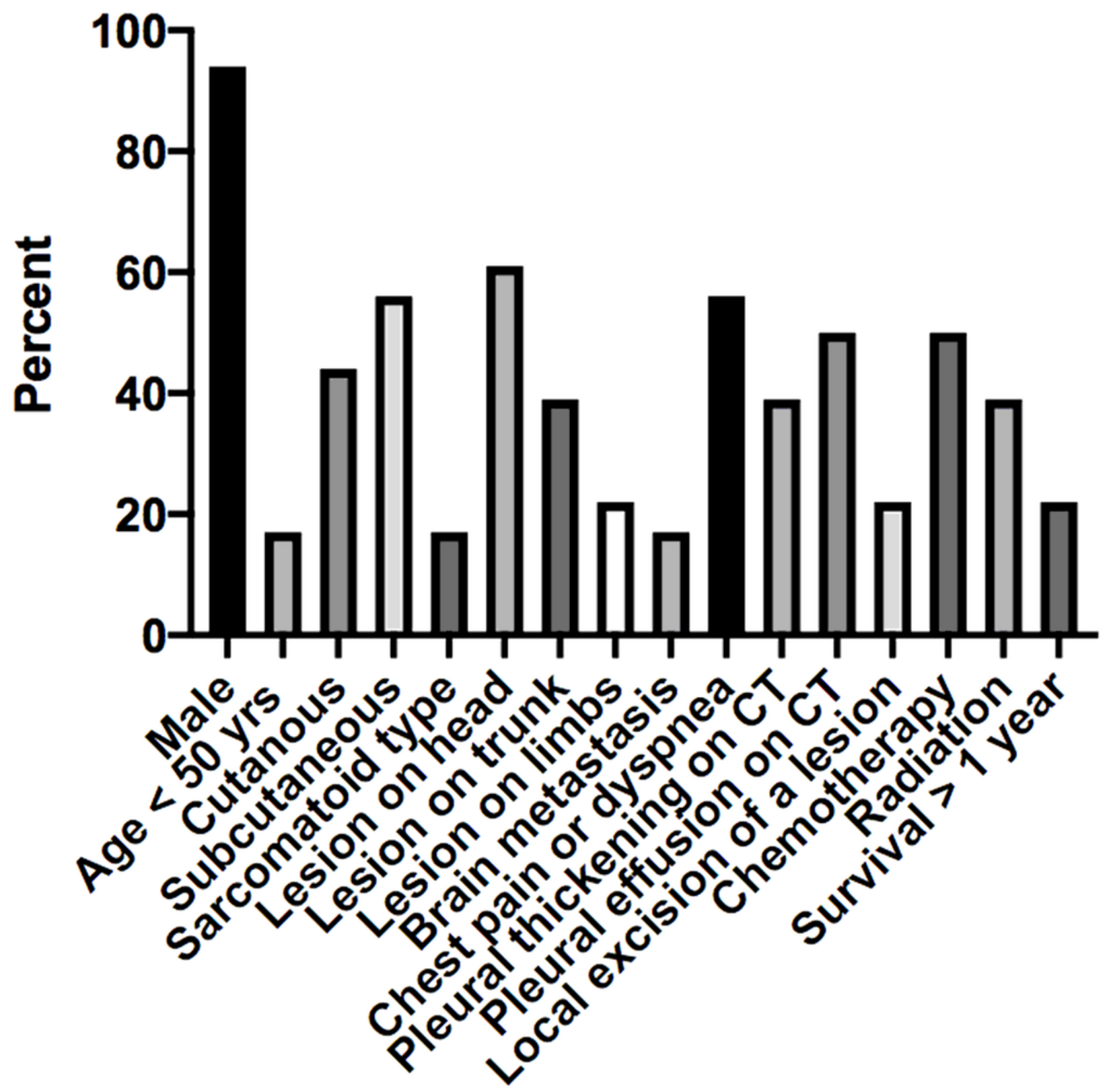

Figure 6

The statistical information of cutaneous or subcutaneous metastases from MM in the previous 18 cases including sex, location, distal metastasis, symptoms, CT features, interventions and prognosis. 


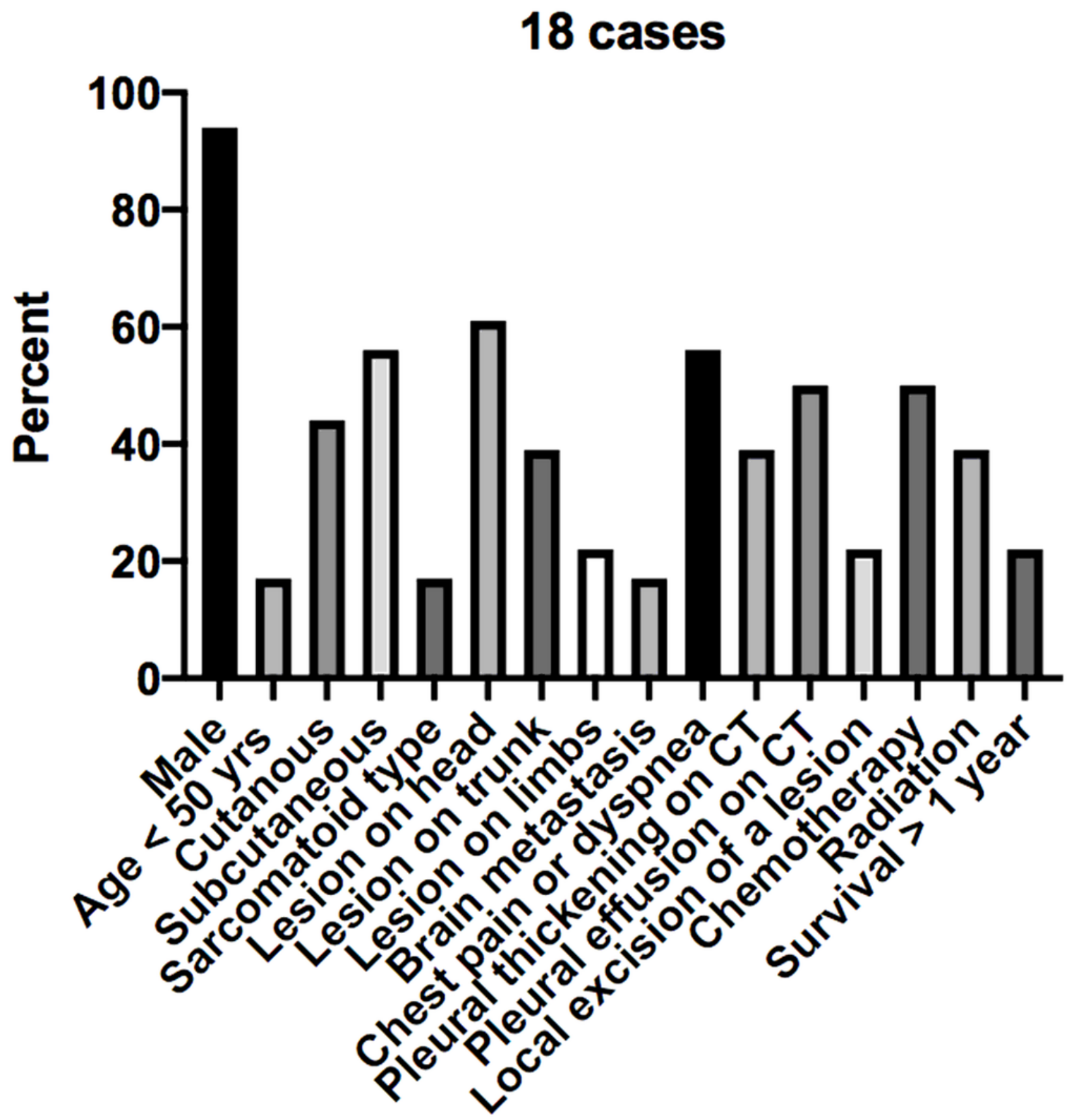

Figure 6

The statistical information of cutaneous or subcutaneous metastases from MM in the previous 18 cases including sex, location, distal metastasis, symptoms, CT features, interventions and prognosis.

\section{Supplementary Files}

This is a list of supplementary files associated with this preprint. Click to download.

- renamed2f5f8.png

- renamed2f5f8.png 